Conclusions We have shown that the vast majority of patients deemed to require preoperative cardiac review can be dealt with efficiently through an MDT meeting, with a resulting reduction in the number of outpatient cardiology reviews and in the optimisation time for anaesthesia. The reduction of 70 patients requiring outpatient cardiology opinion equates to 11 fewer cardiology clinics in a 6 month period; the time taken per patient is shorter in the MDT setting. The $10 \%$ reduction in the number of cardiac investigations requested in the prospective group was contrary to one of our hypotheses. Four patients were entered into the aortic surveillance clinic without their anaesthesia being delayed, as valve surgery was not needed. Cardiology outpatient efficiency was improved as patients were referred with all appropriate investigations already performed. The MDT at the Royal Glamorgan Hospital has changed how the pre-assessment unit obtain a cardiology opinion with demonstrable quality improvements via collaboration between cardiologists and anaesthetists, with improved patient care.

\section{THE IMPACT OF A MULTI-DISCIPLINARY CARE PATHWAY FOR PATIENTS WITH REFRACTORY ANGINA ON PSYCHOSOCIAL OUTCOMES, QUALITY OF LIFE AND MEDICATION}

Kevin Cheng*, Christine Wright, Ranil de Silva. Specialist Angina Service, Royal Brompton Hospital

\subsection{6/heartjnl-2017-311726.95}

Introduction Refractory angina (RA) is a growing clinical problem due to improved survival from coronary artery disease (CAD) and an ageing population. These no option patients experience chronic angina-type pain, in the context of CAD, despite optimal medical and revascularisation therapies. Consequently, management of these patients is challenging and outcomes remain suboptimal. As mortality is no worse, the focus of treatment has shifted to improving symptoms, quality of life (QoL) and psychosocial outcomes.

Methods We undertook a single-centre study of the impact of a multi-disciplinary care pathway (Figure 1) on psychosocial

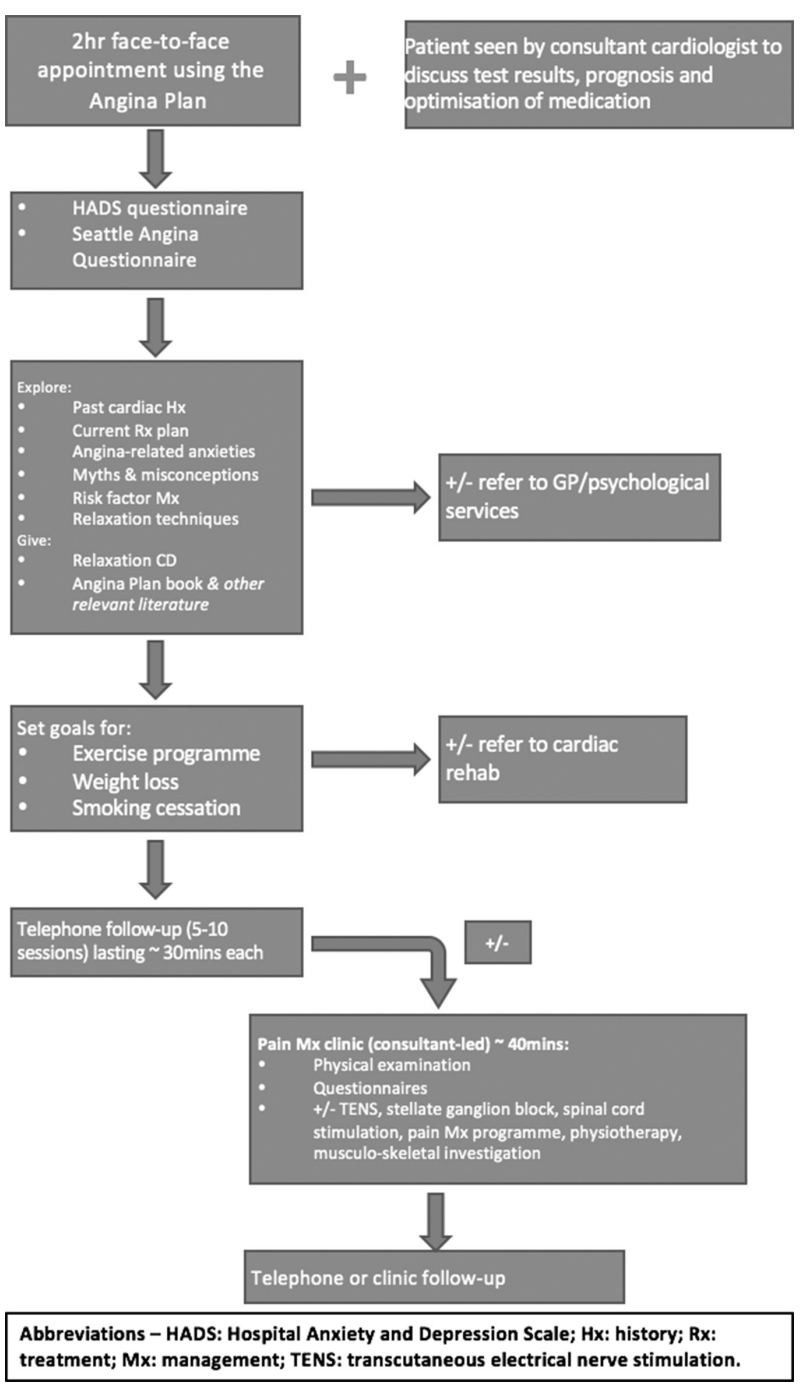

Abstract 96 Figure 1 Schematic showing the multi-disciplinary care pathway for refractory angina at the Royal Brompton Hospital.

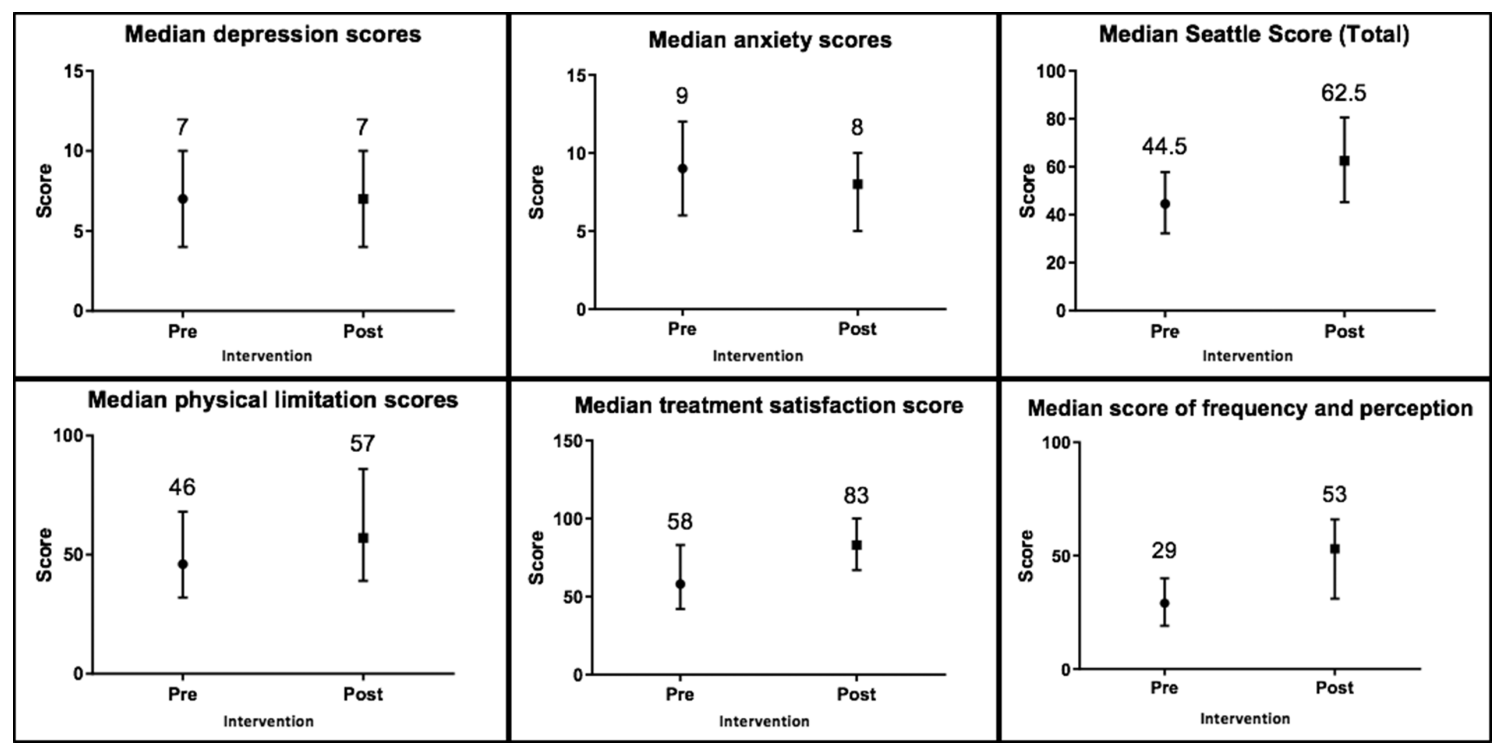

Abstract 96 Figure 2 Graphs showing psychosocial and quality os life scores before after intervention. 
Abstract 96 Table 1 Psychosocial and quality of life outcomes before and after the multi-disciplinary care pathway

\begin{tabular}{|c|c|c|c|c|c|c|c|c|c|c|c|c|}
\hline & \multicolumn{4}{|c|}{ Hospital Anxiety and Depression Scale } & \multicolumn{8}{|c|}{ Seattle Angina Questionnaire score } \\
\hline Outcome & \multirow{2}{*}{\multicolumn{2}{|c|}{ Anxiety }} & \multicolumn{2}{|c|}{ Depression } & \multicolumn{2}{|c|}{$\begin{array}{l}\text { Total (quality of } \\
\text { life) }\end{array}$} & \multicolumn{2}{|c|}{ Physical limitation } & \multicolumn{2}{|c|}{$\begin{array}{c}\text { Frequency \& } \\
\text { perception }\end{array}$} & \multicolumn{2}{|c|}{$\begin{array}{c}\text { Treatment } \\
\text { satisfaction }\end{array}$} \\
\hline No. of patients & & & \multicolumn{2}{|c|}{85} & \multicolumn{2}{|c|}{84} & \multicolumn{2}{|c|}{83} & \multicolumn{2}{|c|}{83} & \multicolumn{2}{|c|}{83} \\
\hline Time point & Pre & Post & Pre & Post & Pre & Post & Pre & Post & Pre & Post & Pre & Post \\
\hline Median score & 9 & 8 & 7 & 7 & 44.5 & 62.5 & 46 & 57 & 29 & 53 & 58 & 83 \\
\hline $\begin{array}{c}\text { Median } \\
\text { difference }\end{array}$ & \multicolumn{2}{|c|}{-1} & \multicolumn{2}{|c|}{-1} & \multicolumn{2}{|c|}{+19} & \multicolumn{2}{|c|}{+8} & \multicolumn{2}{|c|}{+18} & \multicolumn{2}{|c|}{+25} \\
\hline Effect & \multicolumn{2}{|c|}{ Improvement } & \multicolumn{2}{|c|}{ Improvement } & \multicolumn{2}{|c|}{ Improvement } & \multicolumn{2}{|c|}{ Improvement } & \multicolumn{2}{|c|}{ Improvement } & \multicolumn{2}{|c|}{ Improvement } \\
\hline Significance & \multicolumn{2}{|c|}{$p=0.0005$} & \multicolumn{2}{|c|}{$p-0.0469$} & \multicolumn{2}{|c|}{$p<0.0001$} & \multicolumn{2}{|c|}{$p<0.0001$} & \multicolumn{2}{|c|}{$p<0.0001$} & \multicolumn{2}{|c|}{$p<0.0001$} \\
\hline
\end{tabular}

Abstract 96 Table 2 Use of cardiovascular medications before and after the multi-disciplinary care pathway

\begin{tabular}{|c|c|c|c|c|c|c|}
\hline \multirow{2}{*}{$n=72$} & \multicolumn{2}{|c|}{ ON } & \multicolumn{2}{|c|}{ OFF } & \multirow{2}{*}{$\begin{array}{l}\text { \% CHANGE ON } \\
\text { MEDICATION }\end{array}$} & \multirow{2}{*}{ SIGNIFICANCE } \\
\hline & BEFORE & AFTER & BEFORE & AFTER & & \\
\hline$\beta$-blocker & $54(75.0 \%)$ & 47 (65.3\%) & $18(25 \%)$ & 25 (34.7\%) & -9.72 & $p>0.05$ \\
\hline CCB & $45(62.5 \%)$ & $40(55.6 \%)$ & $27(37.5 \%)$ & $32(44.4 \%)$ & -6.94 & $p>0.05$ \\
\hline Long-acting nitrate & $40(55.6 \%)$ & $36(50.0 \%)$ & $32(44.4 \%)$ & $36(50.0 \%)$ & -5.56 & $p>0.05$ \\
\hline Nicorandil & $37(51.4 \%)$ & $28(38.9 \%)$ & $35(48.6 \%)$ & 44 (61.1\%) & -12.5 & $p<0.05$ \\
\hline Ivabradine & $5(6.94 \%)$ & $14(19.4 \%)$ & $67(93.1 \%)$ & $58(80.6 \%)$ & +12.5 & $p<0.0001$ \\
\hline Ranolazine & $7(9.72 \%)$ & $19(26.4 \%)$ & $65(90.3 \%)$ & $53(73.6 \%)$ & +16.7 & $p<0.0001$ \\
\hline ACEI/ARB & $57(79.2 \%)$ & $60(83.3 \%)$ & $15(20.8 \%)$ & $12(16.7 \%)$ & +4.17 & $p>0.05$ \\
\hline Statin & $64(88.9 \%)$ & $60(83.3 \%)$ & 8 (11.1\%) & $12(16.7 \%)$ & -5.56 & $p>0.05$ \\
\hline Antiplatelet & $68(94.4 \%)$ & $68(94.4 \%)$ & $4(5.56 \%)$ & $4(5.56 \%)$ & 0.00 & $p>0.05$ \\
\hline
\end{tabular}

outcomes, QoL and changes in cardiovascular medications. We performed a retrospective search of Electronic Patient Records and databases at the Royal Brompton Hospital for all patients with RA seen between 23/01/2003 and 06/06/2016. Data collected included pre- and post-intervention Hospital Anxiety and Depression Scale and Seattle Angina Questionnaire scores, use of the Angina Plan, referral for specialist pain management and alterations in cardiovascular medications. Median scores and differences for anxiety, depression and QoL were analysed using Wilcoxon matched-pairs signed rank tests. Chisquared tests were used to assess medication changes. Statistical significance was $\mathrm{p}<0.05$.

Results A total of 190 patients with RA were included. The Angina Plan was used in $80.5 \%(n=153)$ and $38.9 \%(n=74)$ patients were referred to pain clinic. Comparison of pre- and post-intervention scores (Table 1) showed significant improvements in anxiety $(p=0.0005)$, depression $(p=0.0469)$, and QoL including physical limitation, frequency and perception of angina symptoms and treatment satisfaction (all $\mathrm{p}<0.0001)$ (Figure 2). Nicorandil use was reduced $(\mathrm{p}<0.05)$ and ivabradine and ranolazine increased $(\mathrm{p}<0.0001)$. Other anti-anginal ( $\tilde{A} f \AA ̊$,-blockers, calcium channel blockers, long-acting nitrates) and disease modifying drugs (angiotensin-converting enzyme inhibitors/angiotensin receptor blockers, statins, antiplatelets) were unchanged $(p>0.05)$ (Table 2).

Conclusions In this study, we showed that a multi-disciplinary care pathway for patients with RA can significantly improve psychosocial outcomes, QoL and medication use. Furthermore, the rate of use of the Angina Plan was high, emphasising the benefits of this specialist nurse-delivered cognitive behavioural therapy-based rehabilitation program in the management of RA. Conventional anti-anginal medication use was largely unchanged or reduced $(52.8 \%$ of patients), in keeping with a non-cardiac aetiology for a proportion of patients symptoms. Ivabradine and ranolazine increased, associated with their introduction to the market. Few data demonstrate the potential impact of specialist services for patients with RA in improving QoL and psychosocial wellbeing, which are the management priorities in this challenging patient group. Our findings suggest that further work in this area is warranted.

\section{CAN PRE-OPERATIVE TROPONIN LEVELS PREDICT POST- OPERATIVE MORTALITY FOLLOWING NON-CARDIAC SURGERY?}

Matthew Jackson*, Mark Hammonds, Nicola Cunningham, Sehrish Khan, Neil Swanson, David Austin, Mark de Belder, Michael Stewart. James Cook University Hospital

\subsection{6/heartjnl-2017-311726.96}

Introduction Despite advances in surgical and anaesthetic techniques, non-cardiac surgery still has a significant mortality. We hypothesised that pre-operative troponin levels might predict post-operative mortality.

Methods Patients undergoing elective and urgent/emergency non-cardiac surgery excluding minor procedures were retrospectively assessed for known vascular disease (defined by diagnostic imaging or previous intervention rather than clinical assessment) and vascular risk factors including hypertension, treatment with lipid-modifying agents (irrespective of agent or dose) and chronic lung disease.

Pre-operative high-sensitivity troponins and routine preoperative bloods were recorded. Six- and twelve-month mortality data were collected; independent predictors of mortality and associations between pre-operative patient characteristics and pre-operative troponin were determined.

Results 993 patients were assessed; 13\% had an elevated preoperative troponin with $3 \%>50 \mathrm{ng} / \mathrm{L} .825$ (83\%) were elective patients; $8.6 \%$ had an elevated pre-operative troponin.

Six-month mortality was $4.2 \%$ and $5.9 \%$ at twelve months. Elevated pre-operative troponin was associated with higher post-operative mortality; $2.5 \%, 12.5 \%$ and $25 \%$ for a troponin $<17 \mathrm{ng} / \mathrm{L}, \quad 17-49 \mathrm{ng} / \mathrm{L}$ and $>50 \mathrm{ng} / \mathrm{L}$ respectively 